\title{
Diacronie
}

Studi di Storia Contemporanea

$\mathrm{N}^{\circ} 30,2$ | 2017

Ponti fra nazioni e continenti

\section{Giorgio Peresso, Giuseppe Donati and Umberto Calosso. Two Italian Anti-fascist Refugees in Malta}

\section{Deborah Paci}

\section{Q OpenEdition}

Edizione digitale

URL: http://journals.openedition.org/diacronie/5921

DOI: $10.4000 /$ diacronie.5921

ISSN: 2038-0925

Editore

Association culturelle Diacronie

Notizia bibliografica digitale

Deborah Paci, « Giorgio Peresso, Giuseppe Donati and Umberto Calosso. Two Italian Anti-fascist Refugees in Malta », Diacronie [Online], № 30, 2 | 2017, documento 9, Messo online il 29 juillet 2017, consultato il 24 septembre 2020. URL : http://journals.openedition.org/diacronie/5921 ; DOI : https://doi.org/ 10.4000/diacronie.5921 


\section{Diacronie}

Studi di Storia Contemporanea

30, 2/2017

Ponti fra nazioni e continenti: diplomazia, immaginari e conoscenze tecniche

\section{RECENSIONE: Giorgio PERESSO, Giuseppe Donati and Umberto Calosso. Two Italian Anti-fascist Refugees in Malta, Gudja, SKS, 2015, $271 \mathrm{pp}$.}

A cura di Deborah PACI

Per citare questo articolo:

PACI, Deborah, «RECENSIONE: Giorgio PERESSO, Giuseppe Donati and Umberto Calosso. Two Italian Anti-fascist Refugees in Malta, Gudja, SKS, 2015, 271 pp.», Diacronie. Studi di Storia Contemporanea : Ponti fra nazioni e continenti: diplomazia, immaginari e conoscenze tecniche, 30, 2/2017, 29/7/2017,

URL: < http://www.studistorici.com/2017/07/29/paci_numero_30/ >

Diacronie Studi di Storia Contemporanea $\rightarrow$ http://www.diacronie.it

Rivista storica online. Uscita trimestrale.

redazione.diacronie@hotmail.it

Comitato di direzione: Naor Ben-Yehoyada - João Fábio Bertonha - Christopher Denis-Delacour - Maximiliano Fuentes Codera Anders Granås Kjøstvedt - John Paul Newman - Deborah Paci - Niccolò Pianciola - Spyridon Ploumidis - Wilko Graf Von Hardenberg

Comitato di redazione: Jacopo Bassi - Luca Bufarale - Gianluca Canè - Fausto Pietrancosta - Alessandro Salvador - Matteo Tomasoni Diritti: gli articoli di Diacronie. Studi di Storia Contemporanea sono pubblicati sotto licenza Creative Commons 3.0. Possono essere

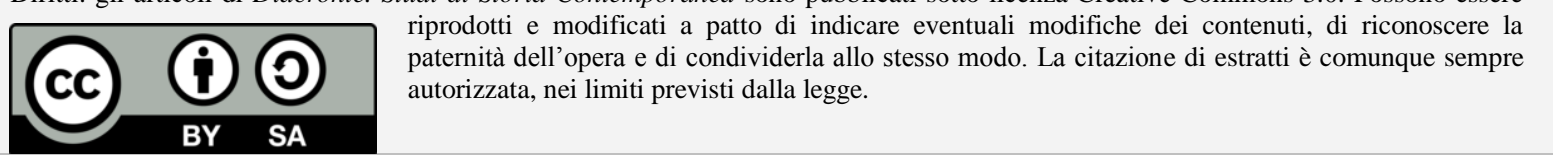




\title{
9/ RECENSIONE: Giorgio PERESSO, Giuseppe Donati and Umberto Calosso. Two Italian Anti-fascist Refugees in Malta, Gudja, SKS, 2015, $271 \mathrm{pp}$.
}

\author{
A cura di Deborah PACI
}

Giuseppe Donati and Umberto Calosso. Two Italian Anti-fascist Refugees in Malta ${ }^{1}$ è la prima monografia di Giorgio Peresso, che trae origine dalla tesi di laurea incentrata sulla vita e l'opera di Giuseppe Donati e Umberto Calosso a Malta, entrambi fuorusciti antifascisti nell'allora arcipelago britannico negli anni Trenta.

Studioso delle relazioni italo-britanniche nel periodo tra le due guerre, Peresso ha conseguito nel 2011 il titolo di dottore magistrale presso l'University of Malta con una tesi intitolata The Enigma of Malta Italica - the saga of irredentism while it lasted. Peresso è inoltre autore di numerosi interventi presentati presso università britanniche tra i quali spiccano, tra i più recenti, When nonbelligerency became belligerent (University of Edinburgh 2014) e Umberto Calosso - Ramingo - the wandering antifascist (University of Reading 2015).

Il libro ricostruisce le traiettorie biografiche di Giuseppe Donati e Umberto Calosso, antifascisti italiani accomunati dall'aver svolto, in periodi distinti, la professione di insegnanti di italiano presso il St. Edward College. Mentre il primo consacrò a Malta gli ultimi nove mesi della sua vita nel 1931, prima di essere colpito mortalmente dalla tubercolosi, Calosso, che lo sostituì nel ruolo di professore di italiano, trascorse ben nove anni nel piccolo arcipelago seppur in maniera intermittente.

Malta fu terra di esilio per eccellenza per gli italiani: basti pensare ad illustri personalità che, come Francesco Crispi e Nicola Fabrizi, scelsero Malta, in epoca risorgimentale, per poter sfuggire alla censura ed esprimere liberamente il proprio pensiero ${ }^{2}$.

\footnotetext{
${ }^{1}$ PERESSO, Giorgio, Giuseppe Donati and Umberto Calosso. Two Italian Anti-fascist Refugees in Malta, Gudja, SKS, 2015.

${ }^{2}$ Ampia è la letteratura sugli esuli italiani a Malta. Si vedano a titolo di esempio ISABELLA, Maurizio, Risorgimento in exile: Italian émigrés and the liberal international in the post-Napoleonic era, Oxford-New York, Oxford University Press, 2009; PACI, Deborah, «Le dialogue des élites méditerranéennes à travers les médias au XIXe siècle: le cas de Malte et de la Corse», Cahiers de la Méditerranée, 43, 85, 2/2012, pp. 11-30; PORTELLI, Sergio, La stampa periodica in italiano a Malta, Msida, Malta University Press, 2010; BONELLO, Vincenzo, FIORENTINI, Bianca, SCHIAVONE, Lorenzo, Echi del Risorgimento a Malta, Malta, Comitato della Società 'Dante Alighieri', 1963.
} 
Il volume si snoda - nelle sue 271 pagine - nel ripercorrere la vicenda umana e pubblica dei due antifascisti che poterono esercitare la professione di insegnanti di italiano in grazia della loro origine italiana, del credo cattolico e della fede antifascista. Difatti tra le caratteristiche richieste per occupare un posto, da assegnare a un madrelingua italiano, al St. Edwards College di Malta vi era quella - oltre ad essere cattolico - di avere un comprovato orientamento antifascista ${ }^{3}$.

Il volume è ripartito in dieci capitoli, una parte dei quali dedicati a Donati, l'altra a Calosso. Nel primo capitolo (A one-man battle against Mussolini ${ }^{4}$ ) l'autore ricostruisce il clima politico degli anni immediatamente successivi al primo dopoguerra; la ricostruzione è affiancata dalla narrazione della vicenda umana e politica di Giuseppe Donati, nato a Granarolo di Faenza il 5 gennaio 1889. Di provata fede cattolica secondo lo spirito dell'enciclica Rerum Novarum, Donati si schierò dalla parte degli interventisti durante il primo conflitto mondiale, avendo concepito la guerra come uno strumento di emancipazione, in senso cristiano, delle classi lavoratrici ${ }^{5}$. Tornato al fronte dopo il matrimonio con Vidya Morici nel 1916, gli fu assegnato l'incarico di giornalista responsabile della propaganda indirizzata ai soldati. In quel frangente lavorò fianco a fianco con Ugo Ojetti ${ }^{6}$, noto giornalista firma del «Corriere della Sera» e assiduo frequentatore dell'arcipelago maltese. Proprio a Ojetti fu attribuita la famosa frase: «i maltesi vogliono la lingua di Dante, la religione di Roma e la sterlina di Londra» ${ }^{7}$. L'avvento del fascismo fu condannato da Donati che fu uno dei pochi giornalisti ad accusare per primo apertamente il governo mussoliniano per la scomparsa - e, in seguito, per il delitto - del deputato socialista Giacomo Matteotti. Questa presa di posizione costò a Donati l'ostilità degli ambienti fascisti, che desideravano sbarazzarsi di lui al più presto; la mediazione dell'allora Ministro dell'interno Luigi Federzoni convinse Donati ad abbandonare l'Italia per trasferirsi in Francia dove girovagò per cinque anni: non gli fu però possibile portare con sé la sua famiglia, che rimase in Italia, potenziale ostaggio delle forze di polizia fasciste ${ }^{8}$.

Il secondo capitolo (Interlude in Malta ${ }^{9}$ ) è dedicato a un approfondimento della condizione del sistema scolastico e dell'insegnamento della lingua italiana nell'arcipelago maltese negli anni Venti. L'allora Primo Ministro maltese Lord Gerald Strickland, che negli anni Trenta avrebbe ingaggiato una strenua lotta contro la lingua e la cultura italiana a Malta opponendosi al suo più

\footnotetext{
${ }^{3}$ PERESSO, Giorgio, op. cit., pp. 33-37.

${ }^{4}$ Ibidem, pp. 3-31.

${ }^{5}$ Ibidem, p. 4.

${ }^{6}$ Ugo Ojetti avrebbe inaugurato l'Istituto Italiano di Cultura a Valletta il 14 febbraio 1932. Cfr. PACI, Deborah, Corsica fatal, Malta baluardo di romanità. L'irredentismo fascista nel mare nostrum (1922-1942), Firenze, Le Monnier-Mondadori Education, 2015, p. 188.

${ }^{7}$ PERESSO, Giorgio, op. cit., p. 6.

${ }^{8}$ Ibidem, p. 24.

${ }^{9}$ Ibidem, pp. 33-84.
} 
ferreo avversario, il nazionalista Enrico $\mathrm{Mizzi}^{10}$, fu intenzionato a rimediare al deficit della cultura britannica nell'arcipelago stabilendo una scuola, il St. Edward College, che potesse fornire agli alunni un'educazione imperniata sui valori etici britannici ${ }^{11}$. Qui la vicenda personale di Donati si interseca con quello dei destini della lingua e della cultura italiana sull'isola. Peresso si sofferma sulla vicenda umana del Donati esule: dopo un periodo di soggiorno a Parigi, decise di trasferirsi a Malta anche per uscire dalla condizione di ristrettezze economiche patite nella capitale francese. L'autore si sofferma poi sulla vita di Donati dall'arrivo a Malta: lo fa soprattutto traendo informazioni dalla corrispondenza epistolare, da cui ricava anche elementi per sondarne lo stato d'animo. Ad esempio Peresso riferisce come il fuoruscito fosse sensibile alle condizioni climatiche e in particolare al vento di scirocco che spirava in tutto l'arcipelago, in grado - a suo giudizio - di condizionarne l'umore ${ }^{12}$.

A Malta Donati dovette fronteggiare il fatto che la difesa dell'italianità fosse divenuta - in un'epoca in cui l'Italia fascista aveva esplicitato le proprie mire sull'isola - quasi conseguentemente l'espressione di sentimenti filofascisti. Ad esempio firmò un articolo pubblicato sul «Malta» il 22 maggio 1931 intitolato Fandonie stricklandesche in cui condannò in maniera risoluta i tentativi da parte di Gerald Strickland di sminuire l'importanza della lingua e della cultura italiana a Malta ${ }^{13}$. Donati si trovò così ad affrontare un "esilio nell'esilio"14, osteggiato da chi guardava con sospetto a quell'italiano che insegnava la lingua che Mussolini avrebbe voluto imporre insieme al suo regime.

L'esule italiano sarebbe morto nel 1931 a Parigi, rientrato in Francia per non concludere i suoi giorni su quello che chiamava «uno scoglio mediterraneo»: la tubercolosi di cui si era ammalato si era infatti aggravata fino all'esito finale. Morì a Parigi il 16 agosto 1931 sepolto nel cimitero di Pantin, dove gli diedero l'ultimo omaggio altri fuorusciti, tra i più noti Carlo e Nello Rosselli, Pietro Nenni, Riccardo Bauer, Ferruccio Parri e Gaetano Salvemini ${ }^{15}$.

Fu Umberto Calosso - a cui sono dedicati tutti i capitoli successivi - a sostituire Donati al St. Edward College. All'anagrafe Umberto Matteo Carlo Calosso, nato a Belveglio d'Asti il 23 settembre 1895, si formò negli ambienti dell'antifascismo torinese (capitolo tre: Another anti-fascist in Malta: Umberto Calosso ${ }^{16}$ ); in contatto con Antonio Gramsci e Piero Gobetti, consacrò la sua vita ad un antifascismo militante e a Malta si impegnò, assieme alla moglie, a disgiungere l'insegnamento della letteratura italiana dal fascismo (capitolo quarto: Umberto Calosso roams in

\footnotetext{
${ }^{10}$ PACI, Deborah, Corsica fatal, Malta baluardo di romanità, cit., pp. 159-167.

${ }^{11}$ PERESSO, Giorgio, op. cit., p. 38.

${ }^{12}$ Ibidem, p. 49.

${ }^{13}$ Ibidem, pp. 65-66.

${ }^{14}$ Ibidem, pp. 51-52.

${ }^{15}$ Ibidem, pp. 71-74.

${ }^{16}$ Ibidem, pp. 87-95.
} 
$\left.M a l t a^{17}\right)$. Tale era il suo profilo di intellettuale che, benché fosse di fatto un "dipendente" di Gerald Strickland, per lui ebbe parole di stima persino l'allora ministro dell'educazione Enrico Mizzi ${ }^{18}$. Calosso si spostò poi in Spagna dove prese parte alla Guerra civile tra le fila dei lealisti repubblicani (capitolo cinque: The freedom fighter in action ${ }^{19}$ ).

Con il rientro a Malta, Calosso si trovò in una situazione ancora più difficoltosa, osteggiato da clerici e filofascisti per il suo impegno attivo durante la guerra di Spagna. Pur avendo ottenuto il passaporto maltese nell'aprile 1940 divenendo a tutti gli effetti suddito della Corona, non poté vedere esaudito il desiderio di occupare il posto di professore di letteratura italiana all'università (capitolo sesto: The quest for the Chair of Italian Literature - a mirage $e^{20}$ ). La sua vicenda, durante il periodo bellico è particolarmente significativa delle peregrinazioni degli antifascisti in questo periodo. Fu dapprima in Tunisia (per organizzare, su proposta del servizio di intelligence del Regno Unito, la propaganda e la pubblicazione di un giornale antifascista in lingua italiana di orientamento filo-britannico), poi a Lisbona con la moglie per nove mesi (impossibilitato a rientrare a Malta dalla possibile imminenza dell'invasione italo-tedesca) e infine in Egitto (come coordinatore della propaganda alleata per gli italiani in Medio Oriente). Proprio nella capitale egiziana Calosso avrebbe maturato l'idea, per la verità ancora prematura, dell'urgenza di creare un fronte comune, sul modello del Fronte popolare spagnolo ma con l'inclusione dei cattolici di Don Luigi Sturzo, da opporre al regime fascista (capitolo settimo: At Tunisia, Lisbon, Egypt and England $\left.^{21}\right)$. Fu anche tra le voci di Radio Londra che, come rileva Peresso, con il suo inconfondibile accento piemontese espresse in modo inequivocabile il suo pensiero antifascista ${ }^{22}$.

Ritornato a Malta dopo la guerra, perse la cittadinanza britannica - acquisita anni prima - a seguito del suo successivo rientro in Italia ${ }^{23}$. Entrò nel Partito socialista dei lavoratori italiani quando questo si scisse dal Partito socialista italiano: non si riconosceva appieno negli ideali e nella linea politica del PSI caratterizzata in quel periodo dalla stretta alleanza con il Partito comunista (capitolo ottavo: Calosso's Republican ideals triumph ${ }^{24}$ ). Divenuto parlamentare, portò avanti una serie di battaglie, soprattutto nel campo dell'istruzione, fino alla sua morte, sopravvenuta il 9 agosto 1959 (capitolo nono: The storm in a teacup ${ }^{25}$ ). Negli ultimi due capitoli (The final act $^{26}$ e Linking Donati and Calosso ${ }^{27}$ ) Peresso rileva come i due fuorusciti, in particolare Calosso,

\footnotetext{
${ }^{17}$ Ibidem, pp. 97-124.

${ }^{18}$ Ibidem, pp. 99-101.

${ }^{19}$ Ibidem, pp. $125-140$.

${ }^{20}$ Ibidem, pp. 141-158.

${ }^{21}$ Ibidem, pp. 159-187.

${ }^{22}$ Ibidem, p. 176.

${ }^{23}$ Ibidem, pp. 190-193.

${ }^{24}$ Ibidem, pp. 189-203.

${ }^{25}$ Ibidem, pp. 205-216.

${ }^{26}$ Ibidem, pp. 217-225.

${ }^{27}$ Ibidem, pp. 229-231.
} 
abbiano lasciato un ricordo positivo presso molti a Malta. Prova ne è la decisione nel 1972 da parte del Primo Ministro laburista Dom Mintoff di intitolare a Calosso una nuova scuola.

Peresso fa ricorso ad una pluralità di fonti primarie, fra cui spicca il costante e minuzioso ricorso all'epistolario dei due antifascisti, avendo avuto accesso alla documentazione rinvenibile presso gli archivi privati e istituzionali in Italia, Gran Bretagna e a Malta. Il libro risulta di particolare interesse sotto differenti punti di vista: è anzitutto un doppio profilo biografico, focalizzato sugli anni trascorsi nell'arcipelago maltese, utile per chi volesse avviare una ricerca complessiva sulla biografia dei due antifascisti. Come Peresso rileva nelle pagine conclusive, i due fuorusciti non tennero lo stesso atteggiamento rispetto alla scena politica maltese: «while Donati in Malta was not anti-British, he defended italianità using the same platform of perceived fascist fellow travellers. Calosso was very pro-British but, while in Malta, he did not participate in openly supporting the agenda of his sponsors» ${ }^{28}$. Il contesto in cui agivano Donati e Calosso è ricostruito in modo da restituire un quadro di insieme ricco e variegato. Questo aspetto ne fa un'opera adatta anche ad un pubblico di non addetti ai lavori. Uno dei pregi maggiori del lavoro di ricerca di Peresso, tuttavia, è dato dalla sua ricostruzione minuziosa degli spostamenti di Donati e Calosso. Le vite di questi due antifascisti divengono un caso di studio per analizzare le condizioni di vita e di azione degli intellettuali europei costretti all'esilio durante gli anni Venti e Trenta. La storia intellettuale tracciata da Peresso - incentrata sul St. Edward College di Malta - si fa così, giocoforza, transnazionale e giunge a cogliere e a narrare dinamiche più vaste e complesse delle singole vicende biografiche. Infine, bisogna considerare la ragione che ha indotto Peresso a occuparsi di queste due figure di antifascisti italiani: il loro incarico di professori d'italiano a Malta. In questa veste dovettero infatti affrontare una condizione di "esilio nell'esilio": esuli dall'Italia per il loro credo politico antifascista, si ritrovarono ad essere guardati con sospetto dai maltesi per via dell'erronea equazione italiano-fascista. A questo non contribuì certamente l'annosa questione linguistica e la lotta politica che vide i due principali schieramenti politici, il partito costituzionale e il partito nazionalista, fronteggiarsi sul terreno della difesa della lingua e della cultura italiana ${ }^{29}$.

A Donati e Calosso spettò - l'uno dopo l'altro, visto che si succedettero nell'incarico - il compito di disgiungere l'insegnamento della lingua italiana dalle mire espansionistiche dell'Italia fascista, riportando - o cercando di riportare - i rapporti tra l'Italia e Malta su di un piano culturale.

Il volume è corredato di un'ingente mole di foto, che accompagnano i singoli paragrafi e svolgono un'utile funzione didascalica. Completano il libro di Peresso tre appendici, alcune pagine

\footnotetext{
${ }^{28}$ Ibidem, p. 231.

${ }^{29}$ HULL, Geoffrey, The Malta language question. A case History in Cultural Imperialism, Valletta, Said International, 1993; PACI, Deborah, Corsica fatal, Malta baluardo di romanità, cit., pp. 22-29.
} 
di note bibliografiche dedicate alle principali personalità trattate nel testo, un apparato bibliografico e un indice dei nomi. 


\section{L'AUTORE}

Deborah PACI è assegnista di ricerca presso il Dipartimento di Studi Linguistici e Culturali Comparati dell'Università Ca' Foscari di Venezia e ricercatrice associata presso il Centre de la Méditerranée Moderne et Contemporaine (CMMC) dell'Université de Nice Sophia Antipolis. Ha conseguito il dottorato di ricerca presso l'Università di Padova in cotutela con l'Université de Nice Sophia Antipolis. È autrice dei libri: L'arcipelago della pace. Le isole Åland e il Baltico (XIX-XXI sec.), Milano, Unicopli, 2016; Corsica fatal, Malta baluardo di romanità. L'irredentismo fascista nel mare nostrum (1922-1942), Firenze, Le Monnier-Mondadori Education, 2015. Ha inoltre curato i volumi: (con L. G. Manenti) Irredentismi. Politica, cultura e propaganda nell'Europa dei nazionalismi, Milano, Unicopli, 2017 e (con E. Grandi) La politica degli esperti. Tecnici e tecnocrati in età contemporanea, Milano, Unicopli, 2014.

URL: < http://www.studistorici.com/progett/autori/\#Paci > 